\title{
Toward a Psychology of Resistance
}

Jeanette A. Lawrence, Melbourne School of Psychological Sciences, The University of Melbourne, Melbourne, Vic 3010, Australia

and

Agnes E. Dodds, Melbourne Medical School, The University of Melbourne,

Melbourne, Vic 3010, Australia

Corresponding author:

Assoc. Prof. Jeanette Lawrence, Melbourne School of Psychological Sciences, Address: Melbourne School of Psychological Sciences, Redmond Barry Building, The University of Melbourne, Melbourne, Vic. 3010, Australia. Email:

lawrence@unimelb.edu.au.

Ph: +61 4 19181624; Fax: +61393476618 


\begin{abstract}
Resistance is all around us in contemporary life. It is an everyday phenomenon of personal and cultural life, as the Chaudhary, Hviid, Marisco \& Villadsen (2017) volume establishes with theoretical analyses and empirical examples from diverse cultural contexts. Resistance functions in the dynamics of person-by-culture encounters when one party takes an opposing position. With the purpose of working towards a unified approach to resistance, the authors in this volume lay down the foundations for a psychology of resistance as an everyday phenomenon. As a basis for analyzing the role of resistance in the dialectical processes of change, the volume presents resistance as: personal and social, oppositional, intentional and future-oriented, transformational and developmental. We see different positions opening up a debate about whether resistance is a particular, intentional and oppositional phenomenon, or is the basic process of all dialectical transformational change. Further resistance to either position is consistent with moving forward in the development of a psychology of resistance.
\end{abstract}

\title{
Key Words
}

Resistance Opposition Intention Internalization Dialectical processes Person-byculture encounters 
Resistance is all around us. It arises in international arenas as disputes about territory, wealth and national interests; in national arenas as clashes between political movements and popular demands for legislative change; and in family as intergenerational disputes and abuse. Resistance arises and persists throughout all levels of people's everyday experiences. It is an ordinary rather than extraordinary phenomenon that occurs in any space and at any time where people oppose social institutions and one another.

People have always resisted institutions, people, and even themselves. Twentyfirst century technology, however, brings public and private occasions of resistance into greater prominence and accessibility. Struggles in India, Egypt and Spain are globally available in daily news. People express their personal struggles and resistances through social media. Commentaries by victims and witnesses of terrorism, family violence, or harassment, for example, when posted on social media lay down traces of resistance as initial acts of opposition for others to join in, react against, or interpret. Resistance is an everyday part of $21^{\text {st }}$ century life, and this volume Resistance in Everyday Life: Constructing cultural experiences (Chaudhary, Hviid, Marisco \& Villadsen, 2017) is both timely and topical. It opens up a dialogue on resistance as a significant sociocultural phenomenon, and raises questions about how a psychology of resistance should be construed. The volume was produced with the related purposes of presenting "the manifestation of resistance in everyday experiences" (Chaudhary et al., 2017, p. 5); and of thus working "towards developing a unified theoretical position" (Chaudhary \& Valsiner, 2017, p. 321). These clearly are big asks, particularly since the individual contributions grew out 
of a workshop involving lively and "highly spiced" (Chaudhary et al., 2017, p. 2) discussions of diverse theoretical positions and empirical examples.

It is significant that the essays and analyzes originated in a workshop in India: the country that Gandhi changed by his new forms of political and cultural resistance. Gandhi's resistance provides the launching point for the diverse cases of public and private life in India and beyond. For example, Pillai (2017) analyzes how Indira Gandhi handled tension throughout her political career, using opposition to create her personal style and national priorities. From a different angle on gender, Sagar and Bhargava (2017) reveal how Dalit ('untouchable') women achieve success in family and cultural life despite multiple disadvantages related to their caste and class. These and other specific cases are given further meaning in Tuli's (2017) analysis of the general cultural changes wrought by Indian women's refusals to conform to cultural and familial expectations. Sunejar and Sharma (2017) similarly invoke the ordinariness of the resistance displayed by Indian children as they navigate and negotiate their ways through and out of culturally expected roles and patterns of behavior. They create their own safe places to play in temples. They disapprove and reinterpret adult meanings for patterns of behavior, acting out subversions of adult-imposed tasks. These examples of the ordinariness and pervasiveness of resistance demand psychological analysis.

The volume, however, is not an analysis of Indian culture. Other empirical examples take the examination into what people do when they are resisting and how transformations follow in diverse cultural arenas (e.g., Egypt, Chile, Europe). More importantly, the empirical examples are wrapped within a set of interpretive essays by the editors who bring different theoretical perspectives to bear on resistance phenomena, for instance, sociocultural, dialectical, psychodynamic. The 
volume thus consciously draws together diverse contributions with the hope of a unified approach on resistance as meaning-making in the dynamics of personal and social engagements, specifically:

The uniting frame in the cultural psychological perspective is the interest in personal meaning-making. Human beings can actively change their environment and things in their environment; they transform them through meaning-making (Chaudhary et al., 2017, p. 6).

The authors agree in asserting the centrality of resistance to human experience. Resistance is "absolutely necessary to human living", because it "guards and nurtures experiences of meaningfulness in living" (Hviid, 2017, p. 14); is "essential to life" in life's regulation and transformation (Marsico, 2017, p.225); "is a vital part of the developmental process" (Villadsen, 2017, p. 120); and is a crucial phenomenon in the interface of people's "dynamic interactions with others" (Chaudhary \& Valsiner, 2017, p. 327).

While this volume goes a long way to establishing the significance of resistance in person-by-culture relations, it appropriately raises questions about the specific role resistance plays in leading to transformational change in these relations. Two themes contribute to the account of resistance under construction. The first establishes the significance of resistance in the everyday dynamics of personal and social life in specific sociocultural contexts. This claim is supported throughout with powerful evidence of resistance across cultures, across sociocultural situations and across the life-course. The second theme focuses on the actual role that resistance plays in dynamic encounters between persons and cultures. This theme raises the theoretical question of whether resistance is the basic driver of all exchanges between persons and social institutions, or whether it is one form of a more basic 
driver in the dialectical process. Does resistance bear the weight of accounting for all person-by-culture encounters and how transformational change proceeds from them, or, does resistance describe a particular type of encounter?

Examining the role of resistance depends, at least in part, on being confident that we can generally agree on the meaning of the concept, without reducing it to a barren psychological construct. We are aware of the danger of distorting contributions made by multiple authors, and propose the following summary account of resistance that is being constructed as a basis for discussing its role in transformational change, and the volume's movement of the field towards a psychology of resistance.

\section{The Account of Resistance Being Constructed}

Awad, Wagoner and Glaveanu (2017) provide a starting place by specifying three characteristics also endorsed in Chaudhary and Valsiner's (2017, p. 325) summary chapter.

... resistance is (1) a social and individual phenomenon; (2) a constructive process that articulates continuity and change; and (3) an act oriented towards an imagined future of different communities. (Awad et al., 2017, p. 161). Working forward from this systematic statement, emphases in other cases and analyses point to resistance as: personal and social; oppositional; intentional and future-oriented; and transformational and developmental.

\section{Personal and social}

As both a personal and social activity, resistance occurs in spaces and times wherever people interact with the sociocultural environment. It is not only personal, because sociocultural institutions also are active participants in the relating, and according to Tateo (2017, p. 233) the relating is crucial - "We live in 
the world and the world lives in us". Individual human systems and sociocultural systems are open to each other as parts of the environment, and they engage in acts of resistance at points of confrontation where the relation is given meaning in what Gottlieb (2007) called 'coactions' between personal and sociocultural systems to emphasize the reciprocal and bidirectional activities involved in their engagements.

Resistance activities are initiated and reacted against in single instances and across inter-connected instances of confrontation in particular sociocultural contexts. It is in the dynamics of these reciprocal and bidirectional actions that resistance has a particular part to play in bringing about systemic change in the person and/or in the cultural collective. Tateo (2017) thus sets the making of meaning in the complementarity of direction and resistance: the "dance of resistance and directionality" (p. 240). The ability of Hijra (transgender) persons to retain their identity in the contemporary world, for instance, can only be understood within the historical and structural context of Indian society. Hijra-bymainstream confrontations and negotiations are imbedded in long-standing patterns of behavior, rituals and fashions that give the Hijra a legitimacy at the margins of social life (Chaudhary \& Shukla, 2017). As it is for Hirja, resistance can be expressed in any modality - physical, intellectual, emotional, but mainly in complex combinations of modalities as they are understood in particular cultural contexts.

\section{Oppositional}

Person-by-culture encounters are resistant when they involve dissent, nonacceptance, conflict, refusal, revolt, disagreement, blockage, that is, any form of opposition. According to Chaudhary et al.'s (2017, p. 3) introduction, resistance is “any form of dissent towards a social phenomenon or social practice by an individual or group". Carré (2017) also shows how the opposition can be to one's 
own intra-personal stances and ideas as they are revised on reflection over time. Diverse cases of this oppositional element are described in terms of Valsiner's (2014) application of 'Gegenstand' (to stand up against) making the active, dissenting aspect of resistance clear, although the example cases speak to the phenomenon of interests arising in specific cultural contexts more clearly than to the underlying explanation of resistance processes. Nevertheless, the oppositional stance is in stark contrast to compliance and conformity and other processes that involve "sharable forms of meaning" (Zittoun, 2009, p. 407). We return to the possibility of non-resistance below.

When a person engages in confrontation with a collective norm, expectation, or practice, and stands against the collective meaning by any activity, the person is resisting oppositionally. A blocking activity represents more than neutral indifference. It expresses something completely other than the original - an antagonistic alternative, making it appropriate to construe resistance processes in terms of the dialectical relations belonging to a phenomenon and its opposite (Marsico, 2017; Villadsen, 2017).

Manifestations of opposition vary with the cultural context and the collective forms of expectation, norm and practice that prevail. They also are likely to vary in the ways of opposing that become open to individuals as they enter confrontational incidents with the particular constraints and affordances of their context. Acts of resistance may seem miniscule and passive from outside the cultural context where they are given meaning in the thinking of the acting agent. For instance, when a garishly clothed Hijra begs for money in the street, an Indian citizen can either respond with a donation or turn away and refuse. How s/he acquiesces or refuses draws on cultural understanding not available to a visiting European. 
Non-acceptance and blockage of possible take-up of the interpersonal into the personal or of the personal into the interpersonal are the marks of resistance, whether or not the opposition is expressed against dominant social forces, as in Awad et al.'s (2017) case of Egyptian political grafitti, or against family expectations that the daughters of Assam tea growers will automatically marry early in the service of the tea tribe (Konwar, Bhargava \& Sharma, 2017).

Opposition is so much a part of resistance, that Marsico (2017) warns against interpreting the dissenting, oppositional dimension exclusively in terms of negative meanings. She like Villadsen, firmly grounds the account of resistance theoretically in dialectical processes, treating oppositional resistance forces as drivers of change. Villadsen adds that the opposition does not belong only to the confrontational event, but also to the conflicting interests that build up to activities within the confrontation that by coaction lead to the re-organization of pieces of the sociocultural landscape, such as when tea grower daughters re-organize their lives and relationships in order to elope and avoid the expected early marriages (Konwar et al., 2017). Opposition works to overcome what exists, making alternatives possible and turning the existing and the possible, into a new actual.

\section{Intentional and future-oriented}

Although inanimate objects are often accused of resisting our human goals, such projections of an essentially human characteristic reflect the intensity of people's frustrations when their intentions are thwarted (Valsiner, 2014). The intentional dimension of resistance is consistently reserved in the volume for descriptions of the conscious activity of a human agent. Intention gives direction and purpose to acts of resistance, when intention formulated in the personal domain is enacted in the interpersonal domain. In whatever way the resistant situation presents and 
evolves, the person's part is guided by the intentions of what s/he values about the self and what s/he wants to achieve for herself and for the community by acting against its unacceptable norm or practice. Intentions shape the person's resisting actions with reference to what s/he imagines can and will happen if s/he opposes a social phenomenon with an oppositional act. The intentional and future-oriented dimensions of resistance are systematically analyzed in Tateo's (2017) treatment of imagination, and are well illustrated by cases of the dynamic formulation and reformulation of resistance activities by Awad et al. (2017) and Carré (2017).

Events that have not yet happened are full of multiple possibilities, even if they are everyday events that recur in relatively stable patterns. As s/he anticipates the next occurrence of a particular type of event, the person is able to mentally run off acceptable and unacceptable scripts of what the other and s/he may do. By imagining these opposing scripts, s/he is able to plan advancing desired happenings and navigating ways through possible constraints. In this anticipatory run-through the event is given personal meaning and direction. Laying out how the processes by which anticipated events can be seen as different future possibilities, Tateo can be said to take up Villadsen's (2017, p. 113) challenge to “elaborate on the processes behind the black box of dialectical leaps". Perceptive imagining gives purpose and direction to anticipated action, and comes with its personalized value tags and priorities.

People interpret objects and events and mentally manipulate them in the service of their goals within the constraints of the particular situation. According to Lewis (2015), such acts of intention can be manipulated only because they exist at the level of awareness and reflection. It may take a member of an oppressed group reflective effort, anticipatory engagement and considerable imagining of 
alternatives before s/he can act out intentional resistance, for example, when a woman anticipates and then first appears without the clothing and facial adornments expected of traditional married women (Tuli, 2017).

Other acts of resistance may be generated on the run within evolving events and lead to modifications of a previously intended activity, as for example, when graffiti artists modified their style or their message in the face of waves of social change in Egyptian cities (Award et al., 2017). Agents act with purpose and act from their intentions, even when these intentions need to be modified or adjusted at the point of confrontation in the given situation.

Personal intention, however, is social as well as future-oriented. The intention remains an incomplete activity until it is expressed in social resistance and brought into cycles of person-by-culture coactions. People do not oppose cultural meanings and practices on all occasions, and do not resist in every confrontation. Their intentions cannot be absolute when other agents are working out their participation simultaneously. A confrontational moment may depend on the subtleties of the coaction where one constraining action grows out of another, for example, when we as university teachers adapted our materials in reaction to students' constraining complaints and where those materials constrained the same students to change their learning strategies (Lawrence \& Dodds, 2007).

Specific instances where resistance is being expressed demonstrate this variability, when resistance is modified to suit overriding environmental pressures. For example, Sharma (2017) describes how Indian adolescents were willing to override personal gratification for the sake of family loyalty in some situations, and in others, to modify their relations to parents to gain the latitude they wanted. The 
directions and intensity of people's intentions in resisting are likely to vary from situation to situation and from person to person.

\section{Transformational and developmental}

Hviid, Villadsen, and Marsico each see resistance as best understood within a dialectical framework that itself stands in opposition to a static, steady-state psychology. Villadsen compares Aristotelian and Hegelian ontologies. Aristotelian inspired, steady-state perspectives do not allow for transformational change. From a dialectical perspective, transformational change is produced when people resist in confrontations, and those coactional confrontations and negotiations are possible because every phenomenon contains within it its own negation. So whenever a norm or practice is asserted or acted-out as that which is in existence and that which should be perpetuated, there also is within its presentation the possibility of its opposite. Someone sees the possibility of 'seeing-the norm-as' (our addition) its alternative, according to Tateo (2017) following Wittgenstein. The tension between positive and negative forces issues in activities that bring about not the original or its negation, but something new and different. Hviid (2017, p. 15) appropriately refers to such culturally contextualized examples of resistance as "developmental occasions" where changes are outcomes for persons and for cultural collectives. Villadsen (2017) reframes Piaget's disequilibration and Erikson's core conflicts as resistances, arguing how these resisting disturbances of the personal system provoke developmental transformations across the life-course. Developmental occasions for change arise in the misfit (Hviid, 2017, p. 14) of the personal and the sociocultural that is an irritant to any smooth running, once the phenomenon and its opposite are seen for what they are and can be. That seeing leads to action, and the reciprocal acting-and-reacting leads to change. 
Villadsen's (2017) quest for the processes involved in these kinds of dialectical leaps takes the discussion into confrontational situations where people contribute to the emergence of new norms and practices by culturally specific resistances. Kapoor, Nagpal and Maggu (2017) illustrate how women's positioning and counterpositioning around gender create new self-definitions and provide the means of challenging and changing traditional family scripts. Their and other empirical examples of activities that lead to dialectical leaps highlight different forms of counter-positioning: negotiations, arguments, silent non-cooperation, subversion. The different forms of transformational change in people's lives in their culture are clear, although the specifically dialectical forces at work are not uniformly as clearly articulated as they are in Tateo's (2017) analysis of imagination.

\section{How Fundamental Is Resistance To Person-By-Culture Encounters?}

Once a dialectical model of transformation and change is followed, it is important to establish the actual role that resistance plays within the dynamic processes. Is resistance as presented in this volume one form of person-by-culture encounter, or is resistance the basic dialectical force in all person-by-culture encounters?

The analyses and examples leave no doubt that "resistance makes development possible" (Chaudhary \& Valsiner, 2017, p. 327). They contain elements of a debate, however, about whether resistance exclusively is the phenomenon that makes development possible and brings about transformational change. A weaker position sees resistance processes as functioning specifically where at least one party acts in oppositional, intentional, future-oriented ways. This position admits the possibility that developmental change may proceed also through other forms of person-byculture exchanges where the receiving person takes cultural meanings into a 
personal system of meaning by internalizing processes that involve transformation along with acceptance (Lawrence \& Valsiner, 2003; Valsiner, 2014).

This position is most clearly articulated in Hviid's chapter, and is given some support by Chaudhary and Valsiner (2017) in their summary chapter. While seeing resistance processes as critical, Chaudhary and Valsiner seem to allow for other possibilities, for instance that, "every encounter is potentially a location for resistance" (p. 321); "Each of these intersection points becomes a potential space of resistance" (p. 325); and "resistance is a crucial phenomenon in this interface" (p. 327). Given that transforming internalization of cultural norms and practices also are admitted as dialectical activities, there may be lee-way here for other possibilities, even to describing resistance as " $\underline{a}$ core of any theory of development" (p. 327, our emphasis).

Hviid (2017) specifically allows for these other dialectical processes by which persons take up, “embrace”, and are "empowered by (these) cultural meanings" (p. 13), reserving resistance for specifically oppositional and intentional phenomenon. She specifically cites Vygotsky's approach and the processes of internalization and appropriation as instances of engagement by which individual persons embrace in their own thinking the cultural meanings held out to them in the sociocultural environment. Processes of enculturation of the young into generally accepted cultural meanings may not always be smooth and certainly not always automatic (Goodnow \& Lawrence, 2015). The person always is presented as agentically active, but not as always taking an intentionally oppositional stance in every encounter with the culture. The processes involved in making the cultural into personal and the personal into cultural are transformational, and Hviid specifically juxtaposes these activities of making the cultural one's own with oppositional resistance 
activities by which persons refuse to admit cultural meanings and stand against the positions held out to them. Important in such personal refusals, as we have discussed above, is the will and intention of the refusing person. This constitutes a special form of dynamic engagement and a particular kind of person-by-culture relational activity.

In contrast, the chapters by Villadsen (2017) and Marsico (2017) lay out for resistance a primary role in the dynamics of dialectical transformation per se. Villadsen also takes a developmental perspective, and like Hviid, refers to how the Vygotskian position is not exclusively directed to resistance. He comments, however, that the Vygotskian tradition "has been commonly criticized for overlooking conflictual aspects of the internalization of collective meanings" (Villadsen, 2017, p. 123).

Marsico (2017), like Villadsen puts resistance at the heart of dialectical processes and of development, serving the origin, transformation and regulation of existence. Here too, resistance is given the fundamental role of "a developing force" (p. 226), for instance, in the origin of life, "providing the minimum indispensable level of structure of any form of life to come into being" (p. 227). There is no room in either of these accounts of the dialectical drivers of change for the accepting, personalizing take-up of the cultural into the personal. The mutuality of the relation between the person and the culture is always oppositional. The parties act out of conflicting interests with the motivation to overcome the other. "Living within a social and cultural organized world incontrovertibly implies living to overcome" (Villadsen, 2017, p. 120). Conflictual interests and intentions to overcome drive the resistance activities and counter-activities that issue in transformational reorganization of the antagonistic parts of the total sociocultural system. It appears 
then that the volume does not present a single, undisputed position on the role resistance plays in person-by-culture encounters, even when it is given a critical role in the dynamics of those encounters.

\section{Conclusion}

With publication of the Chaudhary et al. volume, resistance is established in its rightful place as an everyday phenomenon. It is taken out of the rare happenings category of psychological experiences, and securely entered into the category of ordinary, everyday encounters between persons and their cultural context. Such encounters deserve a place in psychological theory. There is work still to be done in this area, and this volume points the way. Resistance as intentional opposition can be investigated with a $21^{\text {st }}$ Century spotlight. Psychology is less reluctant now to focus on the conscious workings of people's imagined or situationally generated dissenting encounters with cultural forces. The processes of willing take-up and enculturation into cultural meanings need renewed analyses in relation to oppositional resistance, and in relation to how embracing and oppositional processes work towards personal and cultural development. The groundwork has been laid down by Chaudhary et al. In their terms, challenge is to be expected. Whenever scholars set their sights towards any kind of unified approach and present their proposal, albeit a proposal under construction, they are presenting a position that contains within itself alternatives. Resistance is not only to be expected but welcomed in moving forward to bringing resistance into the psychology of everyday life.

\section{Acknowledgements}

Preparation of this paper was supported by the University of Melbourne Schools of Psychology and Medicine. 


\section{Compliance of Ethical Standards}

There is no conflict of interest. No procedures involving human or animal participants were used in the preparation of this paper. The paper complies with the standards of the Australian National Statement on Ethical Conduct in Human Research (2015).

\section{References}

Awad, S. H., Wagoner, B., and Glaveanu, V. (2017). The Street art of resistance. In N. Chaudhary, P. Hviid, G. Marsico, and J. W. Villadsen (Eds.), Resistance in everyday life: Constructing cultural experiences (pp. 161-180). Singapore: Springer.

Carré, D. (2017). Resisting but accepting ideology: Making sense through doublethink. In N. Chaudhary, P. Hviid, G. Marsico, and J. W. Villadsen (Eds.), Resistance in everyday life: Constructing cultural experiences (pp. 133-149). Singapore: Springer.

Chaudhary, N., Hviid, P., Marsico, G., and Villadsen. J. W. (2017). Rhythms of resistance: An Introduction. In N. Chaudhary, P. Hviid, G. Marsico, and J. W. Villadsen (Eds.), Resistance in everyday life: Constructing cultural experiences (pp. 1-9). Singapore: Springer.

Chaudhary, N. and Shukla, S. (2017). The Third gender and their identity in Indian Society. In N. Chaudhary, P. Hviid, G. Marsico, and J. W. Villadsen (Eds.), Resistance in everyday life: Constructing cultural experiences (pp. 35-48). Singapore: Springer.

Chaudhary, N. and Valsiner. J. (2017). Rhythms of resistance and existence: A Way forward. In N. Chaudhary, P. Hviid, G. Marsico, and J. W. Villadsen (Eds.), Resistance in everyday life: Constructing cultural experiences (pp. 319-328). Singapore: Springer. 
Goodnow, J. J., and Lawrence, J. A. (2015). Children and cultural contexts. In M. Bornstein and T. Leventhal (Eds.), Handbook of child psychology and developmental science, Volume 4: Ecological settings and processes in developmental systems. Editor-in-Chief: R. M. Lerner (pp. 746-788). Hoboken, NJ: Wiley.

Gottlieb, G. (2007). Probabilistic epigenesis. Developmental Science, 10(1), 1-11. DOI: 10.1111/j.1467-7687.2007.00556.x

Hviid, P. (2017). Cultural productions through resistance: An Introduction to Section I. In N. Chaudhary, P. Hviid, G. Marsico, and J. W. Villadsen (Eds.), Resistance in everyday life: Constructing cultural experiences (pp. 13-18). Singapore: Springer.

Kapoor, S., Nagpal, V., and Maggu, P. (2017). Culture, gender and resistance: Perspectives from India. In N. Chaudhary, P. Hviid, G. Marsico, and J. W. Villadsen (Eds.), Resistance in everyday life: Constructing cultural experiences (pp. 261-278). Singapore: Springer.

Konwar, D., Bhargava, V., and Sharma, B. (2017). Resisting early marriage - Case study from tea gardens of Assam. In N. Chaudhary, P. Hviid, G. Marsico, and J. W. Villadsen (Eds.), Resistance in everyday life: Constructing cultural experiences (pp. 293-306). Singapore: Springer.

Lawrence, J. A., and Dodds, A. E. (2007). "Myself, the project": Sociocultural interpretations of young adulthood. In J. Valsiner and A. Rosa (Eds.), Cambridge handbook of sociocultural psychology (pp. 404-422). Cambridge: Cambridge University Press. 
Lawrence, J. A., and Valsiner, J. (2003). Making personal sense: An account of basic internalization and externalization processes. Theory and Psychology, 13(6), 723-752.

Lewis, M. (2015). Emotional development and consciousness. In W. F. Overton and P. C. M. Molenaar (Eds.) Handbook of child psychology and developmental science, $7^{\text {th }}$ edn: Vol 1, theory \& method, Editor-in-Chief: R. M. Lerner (pp. 407451). Hoboken, NJ: Wiley.

Marsico, G. (2017). Resistance serves the transformation: An Introduction to Section III. In N. Chaudhary, P. Hviid, G. Marsico, and J. W. Villadsen (Eds.), Resistance in everyday life: Constructing cultural experiences (pp. 225-232). Singapore: Springer.

Pillai, P. (2017). Making meaning out of a lifetime: The Life and times of Indira Gandhi. In N. Chaudhary, P. Hviid, G. Marsico, and J. W. Villadsen (Eds.), Resistance in everyday life: Constructing cultural experiences (pp. 151-159). Singapore: Springer.

Sagar, S. and Bhargava, V. (2017). Dalit women in India: Crafting narratives of success. In N. Chaudhary, P. Hviid, G. Marsico, and J. W. Villadsen (Eds.), Resistance in everyday life: Constructing cultural experiences (pp. 19-33). Singapore: Springer.

Sharma, N. (2017). Adolescent dissent and conflict resolution in the Indian context. In N. Chaudhary, P. Hviid, G. Marsico, and J. W. Villadsen (Eds.), Resistance in everyday life: Constructing cultural experiences (pp. 79-92). Singapore: Springer. Sunejar. S. and Sharma, N. (2017). Children finding their ways through life spaces: Glimpses from the Indian ecology. In N. Chaudhary, P. Hviid, G. Marsico, and J. W. Villadsen (Eds.), Resistance in everyday life: Constructing cultural experiences 
(pp. 65-77). Singapore: Springer.

Tateo, L. (2017). Seeing imagination as resistance and resistance as imagination. In N. Chaudhary, P. Hviid, G. Marsico, and J. W. Villadsen (Eds.), Resistance in everyday life: Constructing cultural experiences (pp. 233-245). Singapore: Springer.

Tuli, M. (2017). Women in Indian families: Resisting everyday. In N. Chaudhary, P. Hviid, G. Marsico, and J. W. Villadsen (Eds.), Resistance in everyday life: Constructing cultural experiences (pp. 307-317). Singapore: Springer.

Valsiner, J. (2014). An Introduction to cultural psychology. London: Sage.

Villadsen, J. W. (2017). A History of resisting the concept of resistance in thinking about human thinking: An Introduction to Section II. In N. Chaudhary, P. Hviid, G. Marsico, and J. W. Villadsen (Eds.), Resistance in everyday life: Constructing cultural experiences (pp. 113-126). Singapore: Springer.

Zittoun, T. (2009). Dynamics of life-course transitions: A methodological reflection. In J. Valsiner, P. Molenaar, M. Lyra, and N. Chaudhary (Eds.). Dynamic process methodology in social and developmental sciences (pp. 405-430). New York: Springer. 